\title{
Human Hair as Adsorbent of Palladium(II) in Solution: A Precursor of Well-Dispersed Size-Controlled Pd Nanoparticles
}

\author{
Fernanda G. Mendonça, ${ }^{a, b}$ Thaís G. Silva,${ }^{a}$ Gustavo M. do Nascimento, ${ }^{c}$ \\ Humberto O. Stumpf, ${ }^{b}$ Raquel V. Mambrini ${ }^{a}$ and Walace D. do Pim ${ }^{\oplus * a}$ \\ ${ }^{a}$ Departamento de Química, Centro Federal de Educação Tecnológica de Minas Gerais, \\ Av. Amazonas, 5253, 30421-169 Belo Horizonte-MG, Brazil \\ ${ }^{b}$ Departamento de Química, Instituto de Ciências Exatas (ICEx), Universidade Federal de Minas Gerais, \\ Av. Antônio Carlos, 6627, 31270-901 Belo Horizonte-MG, Brazil \\ ${ }^{c}$ Centro de Ciências Naturais e Humanas (CCNH), Universidade Federal do ABC, \\ 09210-580 Santo André-SP, Brazil
}

\begin{abstract}
This work describes, for the first time, the preparation of palladium nanoparticles supported in thermally-treated human hair. Human hair showed to be an efficient adsorbent of $\mathrm{Pd}^{2+}$ in aqueous media, reaching nearly $100 \%$ of adsorption from a $100 \mathrm{ppm}$ solution. The thermal treatment of hair containing $\mathrm{Pd}^{2+}$ at $200{ }^{\circ} \mathrm{C}$ under nitrogen atmosphere led to the formation of an $\mathrm{N}, \mathrm{S}$-containing material presenting $0.5 \mathrm{wt}$. \% of palladium. The material was extensively characterized by elemental analysis (CHN and inductively coupled plasma optical emission spectroscopy (ICP OES)), X-ray photoelectron spectroscopy (XPS), Fourier transform infrared (FTIR) and Raman spectroscopies, and by thermogravimetry (TG), thermogravimetry coupled to mass spectrometry (TG-MS), scanning electron miscroscopy (SEM), transmission electron microscopy (TEM) and dispersive X-ray spectroscopy (EDS) analyses. It was possible to observe that the surface structure of hair was preserved during thermal treatment, presenting palladium nanoparticles with particle sizes of approximately $4 \mathrm{~nm}$. This material was used as heterogeneous catalyst in a preliminary application in nitrobenzene reduction to aniline in aqueous medium using sodium borohydride.
\end{abstract}

Keywords: human hair, $\mathrm{Pd}^{2+}$ adsorption, palladium nanoparticles, nitrobenzene reduction

\section{Introduction}

Human hair is a bio-waste or waste biomass composed of lipids and proteins, in which keratin is the main component. It presents essentially carbon, nitrogen, oxygen, hydrogen, and sulfur elements in its composition ${ }^{1,2}$ and is composed by an inner loosely packed region named as medulla surrounded by the cortex, which contains the major part of the fiber mass. The cuticle is the protective layer and makes the outer surface of the fibers. ${ }^{3,4}$

Several research groups have prepared carbonaceous materials derived from human hair, ${ }^{2,5-11}$ which can be used as environmental friendly electrodes with good chemical/ thermal stability, acting in catalysis and electrocatalysis, ${ }^{5-7}$ gas adsorption, ${ }^{2}$ electrochemical sensing, ${ }^{8}$ and as electrochemical supercapacitors. ${ }^{8,10,11}$ For these purposes,

*e-mail: walacedopim@cefetmg.br the pyrolysis temperature must be in the range of 600-900 ${ }^{\circ} \mathrm{C}$ ensuring the formation of graphitic carbon structures containing a small amount of heteroatoms. ${ }^{8,9,11}$

Cysteine and methionine amino acids present in hair fibers are able to interact with soft transition metal ions (e.g. $\mathrm{Ag}^{+}, \mathrm{Au}^{+}, \mathrm{Pt}^{2+}, \mathrm{Pb}^{2+}$, and $\left.\mathrm{Hg}^{2+}\right)$ through sulfur donor atoms in aqueous solution forming metal complexes in the surface of hair fibers. ${ }^{12}$ The same behavior is observed for oxygenated and nitrogenated terminal groups (e.g. arginine, tryptophan, and tyrosine), which can form chelate complexes with hard metal ions such as $\mathrm{Co}^{3+}, \mathrm{Fe}^{3+}$, and $\mathrm{Cr}^{3+}$ for instance. ${ }^{1,13}$ Indeed, hair is a very efficient adsorbent for organic pollutants and metal ions in wastewaters, ${ }^{14-16}$ being utilized as a suitable platform to prepare supported catalytic nanoparticles. ${ }^{17-21}$ The great adsorption properties of human hair were exploited in this work, for the first time, as a previous step in the preparation of low temperature thermal treated hair containing well-distributed palladium nanoparticles. 
Haveli et al. ${ }^{22}$ described the synthesis of gold nanoparticles (AuNPs) inside human hair in a remarkably regular pattern, opening an area of genuine nanocomposites with novel properties due to the AuNPs inside the human hair shaft. Human hair fibers have also been employed as a precursor of doped carbon, which acts as a support for immobilization of metal oxides such as mixed-valence spinel cobalt oxide $\left(\mathrm{Co}_{3} \mathrm{O}_{4}\right)$ nanocrystals. ${ }^{10}$ This feature can improve the energy storage performance of the material, as the highly conductive carbonaceous matrix can increase the electrical conductivity, preventing the aggregation of active cobalt(III) oxide. These materials were produced using $\mathrm{NaBH}_{4}$ as a reduction agent or under extreme conditions of pressure and temperature (hydrothermal synthesis).

In this work, hair fibers were used as adsorbents of $\mathrm{Pd}^{2+}$ ions in aqueous solution. In a second step, the resultant Pd-containing hair fibers were pyrolyzed at mild conditions, e.g. $200^{\circ} \mathrm{C}$, in order to reduce $\mathrm{Pd}^{2+}$ ions, and prevent heteroatoms of organic functional groups from decomposition, ${ }^{23}$ obtaining Pd nanoparticles (PdNPs) well dispersed in the matrix. This procedure is a facile route to prepare supported PdNPs, which are materials of great interest in the field of heterogeneous catalysis. Palladium is a metal employed in a series of catalyzed reactions, especially in fine and environmental chemistry, such as hydrogen generation from formic acid, ${ }^{24}$ isomerization of allyl alcohol,,${ }^{25}$ carbon-carbon coupling, ${ }^{26}$ oxidation of alcohols and semihydrogenation of alkynes, ${ }^{27}$ selective hydrogenation of alkenes ${ }^{28,29}$ and nitroarene compounds. ${ }^{30}$ The unique nature of palladium as a selective catalyst using extremely small amounts of this metal under mild conditions is one of the main reasons that attract researchers. ${ }^{31}$ As a result, a great number of palladium catalysts are commercially available. Since aromatic amines have an enormous application in several condensation reactions for industrial and scientific purposes, the development of green and low-cost heterogeneous catalysts able to act in nitroarene compounds reduction is relevant. Herein is illustrated a preliminary application of the obtained material in nitrobenzene reduction to aniline in aqueous medium.

\section{Experimental}

\section{Materials and characterization}

Hair was collected from a healthy volunteer. Ethanol P.A., $\mathrm{K}_{2} \mathrm{PdCl}_{4}$ were purchased from Sigma-Aldrich. Nitrobenzene was purchased from Merck. Atomic absorption analyses were performed in a spectrometer Hitachi-Z8200 coupled to a Hitachi graphite furnace. Thermogravimetric-mass spectrometry analyses (TG-MS) were performed in a NETZSCH equipment model STA 449 F3, coupled to a mass spectrometer NETZSCH Aëolos model QMS 403C, using argon as carrier gas. The infrared spectra of the materials were obtained using a Fourier transform infrared spectrophotometer (FTIR; Shimadzu, Model IRPrestige-21) by the attenuated total reflectance (ATR) technique. Elemental analyses (CHN) were performed with a PerkinElmer 2400 series II analyzer. Inductively coupled plasma optical emission spectrometry (ICP OES) was performed in a spectrometer Spectro, model Arcos, with radial configuration (SOP) for sulfur determination. FT-Raman spectra of powered samples were recorded in a Bruker RFS 100/S FT-Raman spectrometer using $1064.0 \mathrm{~nm}$ laser line. The data were acquired under room temperature (near $20^{\circ} \mathrm{C}$ ) with the samples supported over a glass microscope slide in a backscattering geometry. The laser beam with approximately $1 \mathrm{~mm}$ spot was focused on the sample and the laser power was changed from 10 to $70 \mathrm{~mW}$ in order to maximize the signal. Raman spectra recorded at $532 \mathrm{~nm}$ laser line (Coherent Inc.) using ca. $20 \mathrm{~mW}$ was obtained in a Horiba JobinYvon T6400 coupled with CCD (charge coupled device) detector in a microscopic mode having $10 \times$ lens. The X-ray photoelectron spectroscopy (XPS) measurements were performed in a Thermo Fisher Scientific, model $\mathrm{K}$-alpha+done by using characteristic $\mathrm{K} \alpha$ radiation from an $\mathrm{Al}$ anode to excite the samples and a $180^{\circ}$ double focusing hemispherical analyser-128-channel detector. A small quantity of each sample was pressed between two stainless steel plates to form a thin conglomerate fixed to the sample holder with double-faced conducting tape. The analyses were done at a base pressure of $5 \times 10^{-9} \mathrm{mbar}$, and charging effects were corrected by shifting the spectra so that the $\mathrm{C} 1 \mathrm{~s}$ line was at $284.6 \mathrm{eV}$. Thermogravimetric analyses (TG/DTA (differential thermal analysis)) were carried out in a Shimadzu TGA-60 equipment $\left(10^{\circ} \mathrm{C} \mathrm{min}^{-1}\right.$, under air flow). Scanning electron microscopy (SEM) images were obtained by using a FEG-Quanta 200 FEI microscope. The samples were suspended in acetone and deposited onto silicon chip specimen support. Transmission electron microscopy (TEM) images were obtained using a Tecnai G2 200 kV SEI microscope. Energy dispersive spectroscopy (EDS) was performed on the same equipment. The samples were suspended in acetone and deposited onto a holey carbon film on 200 mesh copper grids.

\section{Adsorption of metal ions in hair fibers}

Human hair was extensively washed with ethanol and dried at $60{ }^{\circ} \mathrm{C}$. After cleaning, hair was cut in small pieces (5 to $10 \mathrm{~mm}$ long) and was put in contact with the 
$\mathrm{Pd}^{2+}$ solution (100 ppm) and left under shaking for $24 \mathrm{~h}$ at $190 \mathrm{rpm}$. A ratio of $20 \mathrm{mg}$ of hair $\mathrm{mL}^{-1}$ of solution was used at $\mathrm{pH}$ ca. 5.5. The mixture was then centrifuged and the hair was separated from the supernatant. Atomic absorption spectroscopy was used to quantify the amount of remaining $\mathrm{Pd}^{2+}$ in solution.

Synthesis of thermally treated hair fibers with adsorbed $\mathrm{Pd}^{2+}(\mathrm{Pd} / \mathrm{TTH})$

After drying at $60{ }^{\circ} \mathrm{C}$, hair fibers with adsorbed $\mathrm{Pd}^{2+}$ were thermally treated in a horizontal furnace under $\mathrm{N}_{2}$ atmosphere $\left(50 \mathrm{~mL} \mathrm{~min}^{-1}\right)$ with a heating rate of $10^{\circ} \mathrm{C} \mathrm{min}^{-1}$ until $200{ }^{\circ} \mathrm{C}$, keeping this temperature for $1 \mathrm{~h}$. The dark solid obtained was ground to powder and characterized. The elemental analyses showed that $\mathrm{Pd} / \mathrm{TTH}$ is composed of $46.10 \% \mathrm{C}, 5.90 \% \mathrm{H}, 13.74 \% \mathrm{~N}$, and $3.06 \% \mathrm{~S}$.

\section{Catalytic performance of $\mathrm{Pd} / \mathrm{TTH}$ towards nitrobenzene reduction}

In a typical run, $10 \mathrm{~mL}$ of nitrobenzene aqueous solution (100 ppm), $100 \mu \mathrm{L}$ of $\mathrm{NaBH}_{4}\left(0.048 \mathrm{~mol} \mathrm{~L}^{-1}\right)$, and $\mathrm{Pd} / \mathrm{TTH}$ catalyst $(20 \mathrm{mg}$ ) were mixed and the suspension was magnetically stirred. The monitoring of the reduction kinetics of nitrobenzene was performed using a UV-Vis spectrophotometer (Varian Cary 50 Conc), through scanning mode. The concentrations were obtained from calibration curves constructed using the absorbance of nitrobenzene and aniline at 275 and $229 \mathrm{~nm}$, respectively. Reuse studies of Pd/TTH material were carried out under similar conditions. At the end of the reaction, the catalyst was removed from the reaction mixture by centrifugation and used in the next catalytic run without purification, totaling four cycles.

\section{Results and Discussion}

Human hair was put in contact with a $\mathrm{Pd}^{2+}$ solution, originally presenting brown coloration. After being kept in contact with the hair for $24 \mathrm{~h}$ under stirring, the solution became colorless (see Figure S1 in Supplementary Information (SI)), the first evidence of $\mathrm{Pd}^{2+}$ adsorption by hair. A later evaluation through atomic absorption spectroscopy showed that $\mathrm{Pd}^{2+}$ adsorption reached nearly $100 \%$ under the experimental conditions, resulting in a material which contains $0.5 \mathrm{wt}$.\% of Pd. A first attempt to understand the interaction of metal ions with hair fibers can be related to the isoelectric point of human hair, which is observed at approximately $\mathrm{pH} 4 .{ }^{32} \mathrm{As}$ adsorption experiment was performed at $\mathrm{pH}$ value ca. 5.5 , hair fibers will be deprotonated in this condition, indicating that an electrostatic interaction can initially act as driving force for $\mathrm{Pd}^{2+}$ adsorption by human hair.

After $\mathrm{Pd}^{2+}$ adsorption, the material was dried at $60{ }^{\circ} \mathrm{C}$ and named as $\mathrm{Pd} / \mathrm{H}$. This material was thermally treated at $200{ }^{\circ} \mathrm{C}$ under $\mathrm{N}_{2}$ atmosphere and a black powder was obtained, named as Pd/TTH, with 70\% yield. A thermal treatment was also performed with pure hair fibers, in the same conditions, producing the material TTH. The temperature of pyrolysis was fixed at $200{ }^{\circ} \mathrm{C}$ in order to prevent the agglomeration of reduced PdNPs, avoiding the complete degradation of amino acid groups present in hair fibers, without the needing of organic solvents or strong reducing agents. Thus, TG-MS analyses were performed to monitor the loss of sulfur present in human hair, since it is the main atom in hair capable to interact with soft metals as Pd. The sulfur loss was monitored through the evolution of $\mathrm{H}_{2} \mathrm{~S}(\mathrm{~m} / \mathrm{z} 34)$ during the heating of pure hair fibers and $\mathrm{Pd} / \mathrm{TTH}$ (Figure 1a). The shift in the peak observed for hair fibers containing $\mathrm{Pd}^{2+}$ suggests that this metal ion interacts with sulfur-containing amino acids in hair (Figure 1b) and that the loss of these atoms occurs at temperatures slightly higher than $200{ }^{\circ} \mathrm{C}$.

FTIR spectroscopy was used to verify the presence of organic functional groups in pure human hair fibers, TTH, and $\mathrm{Pd} / \mathrm{TTH}$ samples (Figure 1c). It is possible to observe that the IR spectra profile of hair fiber is very similar to the materials treated at $200{ }^{\circ} \mathrm{C}$, TTH, and Pd/TTH. This observation agrees with Zhao et al. ${ }^{2}$ in which hair samples were pyrolyzed at higher temperatures such as $550-650^{\circ} \mathrm{C}$, maintaining the bands referring to the functional groups assigned to different amino acid residues. The bands observed in the range of 3480-3515 and 3265-3240 $\mathrm{cm}^{-1}$ are assigned to $\mathrm{O}-\mathrm{H}$ and $\mathrm{N}-\mathrm{H}$ vibration modes, respectively. ${ }^{33}$ The bands between $2967-2849 \mathrm{~cm}^{-1}$ can be assigned to $\mathrm{C}-\mathrm{H}$ stretching mode due to the presence of several amino acid fragments in the materials. Typical $v \mathrm{C}=\mathrm{O}$ bands observed in the range of $1684-1622 \mathrm{~cm}^{-1}$ are attributed to amide and carboxylic groups in the surface of the thermal-treated hair. ${ }^{34}$ The vibration modes of $\mathrm{C}=\mathrm{C}$ and $\mathrm{C}=\mathrm{N}$ groups can be observed in the range of $1558-1510 \mathrm{~cm}^{-1}$. Sulfur-containing groups were observed through the bands centered at 1171 and $1014 \mathrm{~cm}^{-1}$, assigned to vibrations of $-\mathrm{SO}_{3}{ }^{-}$and $\mathrm{S}=\mathrm{O}$, respectively. ${ }^{34,35}$

The elemental analyses $(\mathrm{C}, \mathrm{H}, \mathrm{N}, \mathrm{S}, \mathrm{Pd})$ of the human hair and the materials TTH and Pd/TTH are presented in Table 1. It was observed an increase in the relative carbon content for the materials obtained after thermal treatment. It is interesting to observe that nitrogen and sulfur contents did not suffer great variations when compared to human hair, confirming the hypothesis that the amino acids groups 


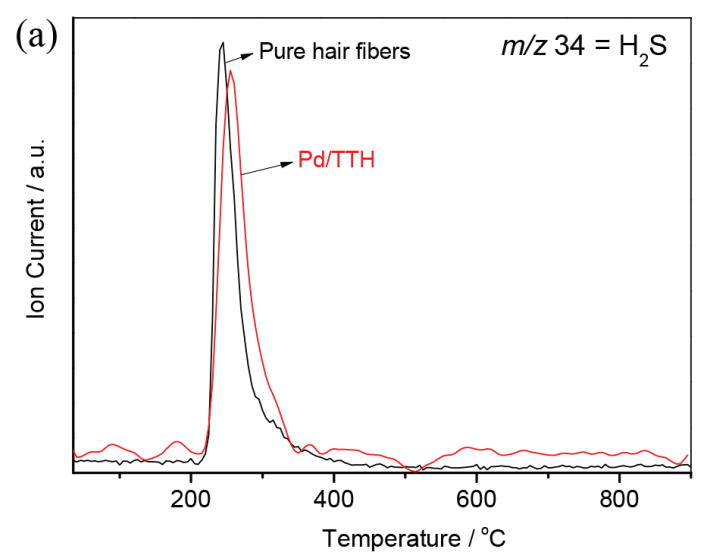

(b)

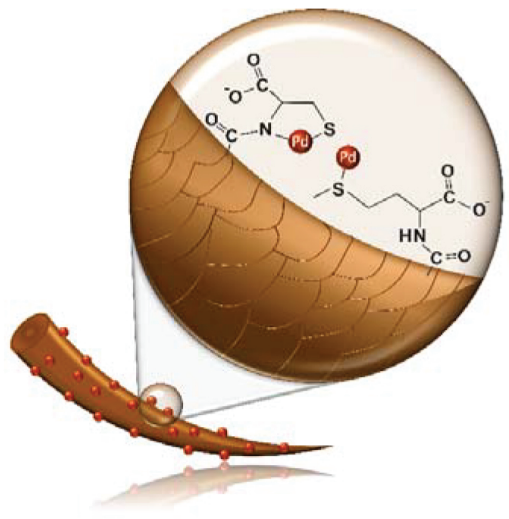

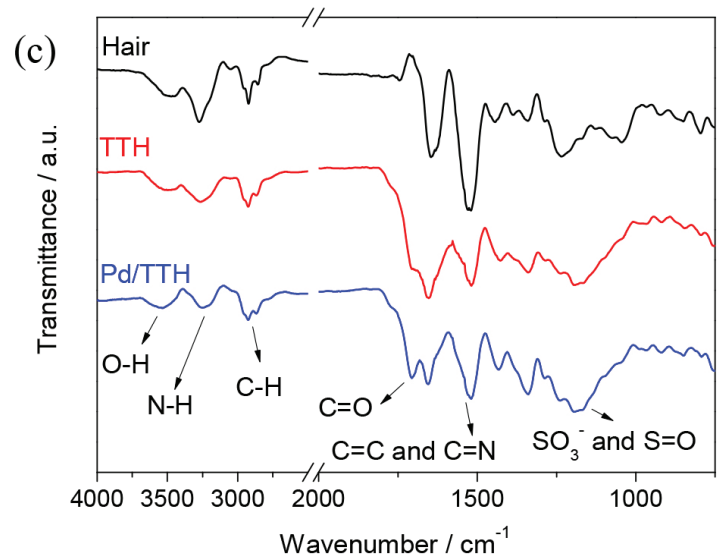

Figure 1. (a) TG-MS curves obtained for pure hair fibers and Pd/TTH samples, monitoring the $\mathrm{m} / \mathrm{z} 34$; (b) schematic representation of the interaction of $\mathrm{Pd}^{2+}$ with sulfur-containing amino acids in hair; (c) FTIR spectra obtained for pure hair fibers, TTH and Pd/TTH samples.

Table 1. Carbon, hydrogen, nitrogen, sulfur, and palladium contents for human hair, TTH, and Pd/TTH samples. Percentage of others was calculated by the difference

\begin{tabular}{|c|c|c|c|c|c|c|}
\hline Material & $\mathrm{C} / \%$ & $\mathrm{H} / \%$ & $\mathrm{~N} / \%$ & $\mathrm{~S} / \%$ & $\mathrm{Pd} / \%$ & Others / \% \\
\hline Human hair & 41.42 & 6.76 & 13.12 & 4.24 & - & 34.46 \\
\hline TTH & 48.68 & 5.99 & 13.92 & 4.54 & - & 26.87 \\
\hline $\mathrm{Pd} / \mathrm{TTH}$ & 46.10 & 5.90 & 13.74 & 3.06 & 0.5 & 30.7 \\
\hline
\end{tabular}

TTH: thermally treated human hair; Pd/TTH: thermally treated hair fibers with adsorbed $\mathrm{Pd}^{2+}$.

were not lost during thermal treatment. The slight increase in the relative sulfur content observed for TTH is due to the dehydration that takes place during thermal treatment, confirmed by a decrease in hydrogen content.

Figure 2 exhibits the Raman spectra obtained for pure human hair fibers, TTH, and Pd/TTH samples. At $1064.0 \mathrm{~nm}$, the bands at 2546, 1591 and $1277 \mathrm{~cm}^{-1}$ observed for hair sample can be assigned to $\mathrm{VS}-\mathrm{H}$ mode, amide I, and collagen I groups, respectively. ${ }^{36}$ In addition, the bands at 1094 and $560 \mathrm{~cm}^{-1}$, assigned to $\mathrm{vSi}-\mathrm{O}$ modes, ${ }^{37}$ are related to the glass slide used for supporting the samples (see the spectrum of glass slide for comparison). All Raman bands associated to hair are not observed in spectra for TTH and
$\mathrm{Pd} / \mathrm{TTH}$ samples at $1064 \mathrm{~nm}$ laser line, probably due to the mild carbonization treatment used in these samples there is no formation of graphitic phase. However, at $532 \mathrm{~nm}$, only for Pd/TTH sample, the Raman spectrum showed large bands at ca. 1190 and $1590 \mathrm{~cm}^{-1}$ that can be related to the $\mathrm{D}$ (disorder and defects) and $\mathrm{G}$ (graphitic $\mathrm{v}_{\mathrm{C}-\mathrm{C}}$ modes) bands characteristic of the carbon allotropes, as in the graphite (Figure 2, inset). The low intensity of these bands and the fact that those bands are Raman resonant at $532 \mathrm{~nm}$ laser line could suggest to the formation of a small number of particles having graphitic structures. This result is coherent with the literature ${ }^{8}$ because only higher carbonization temperatures lead to the formation of graphitic structures. At last, the 
Raman spectra for Pd/TTH and TTH samples showed strong photoemission at $1064 \mathrm{~nm}$, particularly the $\mathrm{Pd} / \mathrm{TTH}$ sample is very sensitive to laser power (see Figure 2). Probably, the presence of PdNPs increases the photoemission process.

TG analysis of Pd/TTH under air atmosphere (see Figure S2 in SI section) showed a significant weight loss starting at $300{ }^{\circ} \mathrm{C}$, related to oxidation of functional groups

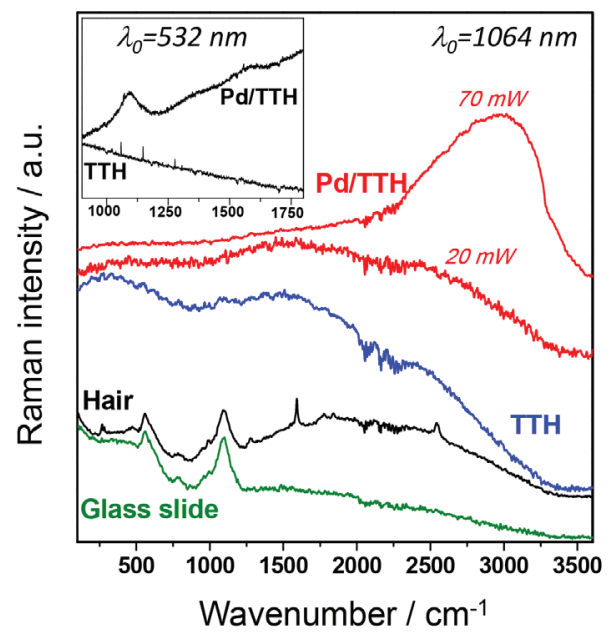

Figure 2. FT-Raman spectra for powered samples of Pd/TTH, TTH and pure hair fibers obtained at $1064.0 \mathrm{~nm}$. For comparison purposes, the spectrum of the glass slide used for supporting the samples was also displayed. Inset: Raman for TTH and Pd/TTH at $532 \mathrm{~nm}$.
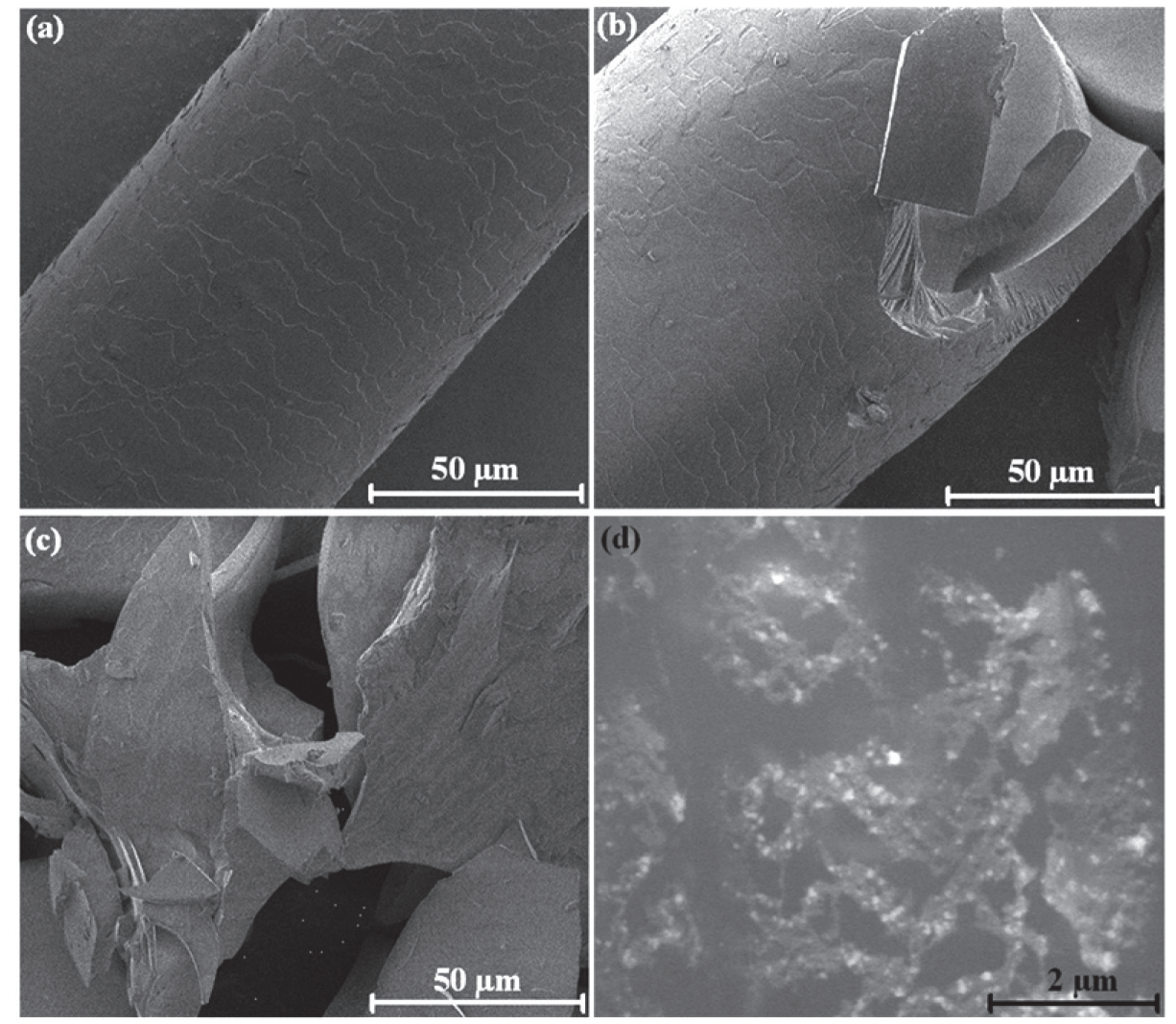

Figure 3. SEM images of (a) pure hair fibers, (b) TTH and (c,d) Pd/TTH. Images (a), (b) and (c) were acquired with secondary electrons and (d) using backscattering electrons. and carbon oxidation. TG curve also showed a slight weight gain at temperatures higher than $600{ }^{\circ} \mathrm{C}$, which is probably

Figure 3 shows SEM images obtained for human hair fibers, TTH and Pd/TTH samples. In the image obtained for human hair (Figure 3a), it is possible to observe the hair cuticle, formed by scales. After pyrolysis, a hollow and brittle carbon is obtained, ${ }^{8,9}$ as observed in Figures $3 \mathrm{~b}$ and $3 c$. Hair cortex and medulla are eliminated, remaining only the cuticle, which maintains its format on the carbon surface. Figure $3 \mathrm{~d}$ shows the image obtained for $\mathrm{Pd} / \mathrm{TTH}$ through backscattering electrons detection, where the lightest points are probably related to the presence of $\mathrm{Pd}^{0}$ in this material.

The presence of $\mathrm{Pd}$ nanoparticles was confirmed by TEM (Figure 4a and Figure S3 in SI section). It is possible to observe for $\mathrm{Pd} / \mathrm{TTH}$ the presence of darker spheres in a carbon matrix, possibly referent to PdNPs highly disperse on carbon. EDS technique also detected the presence of $\mathrm{Pd}$, as shown in Figure $4 \mathrm{~b}$, presenting particles sizes of approximately $4 \mathrm{~nm}$.

The formation of Pd nanoparticles can also be seen in XPS spectra. The binding energies (BE) of the $\mathrm{Pd} 3 \mathrm{~d}_{5 / 2}$ and $3 \mathrm{~d}_{3 / 2}$ electrons in the high-resolution spectrum (Figure 5) appear at 335.7 and $340.7 \mathrm{eV}$, respectively. These values are consistent with the formation of $\mathrm{Pd}^{0} \mathrm{NPs}$ on $\mathrm{TTH}$ related to the oxidation of PdNPs present in this sample. 


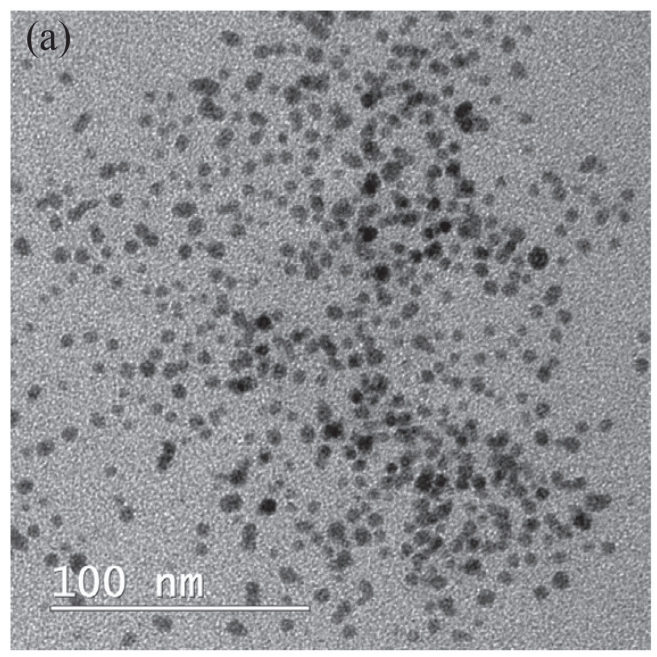

(b)

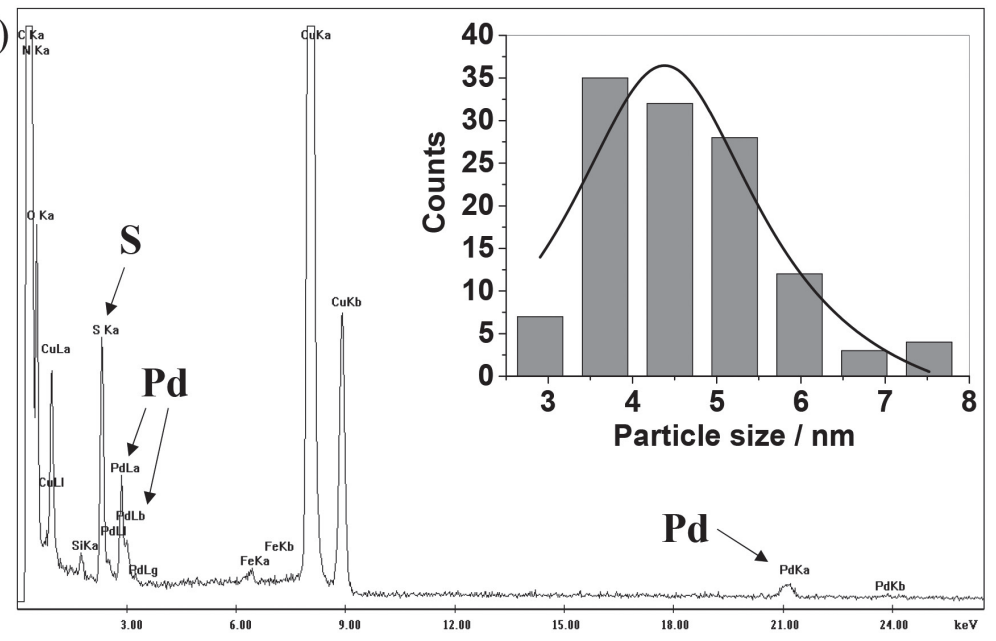

Figure 4. (a) TEM image of Pd/TTH. Darker circles confirm the presence of Pd in this material; (b) EDS spectrum obtained for Pd/TTH, inset: size distribution histogram of PdNPs in Pd/TTH, calculated using ImageJ.

matrix. ${ }^{38}$ In fact, according to Zhou et al..${ }^{39}$ it is possible to correlate the $\mathrm{BE}$ values to the size of Pd nanoparticles. Considering this trend, it is expected that PdNPs prepared here have a size below $9 \mathrm{~nm}$, in fact, this is in accordance to the TEM analysis.

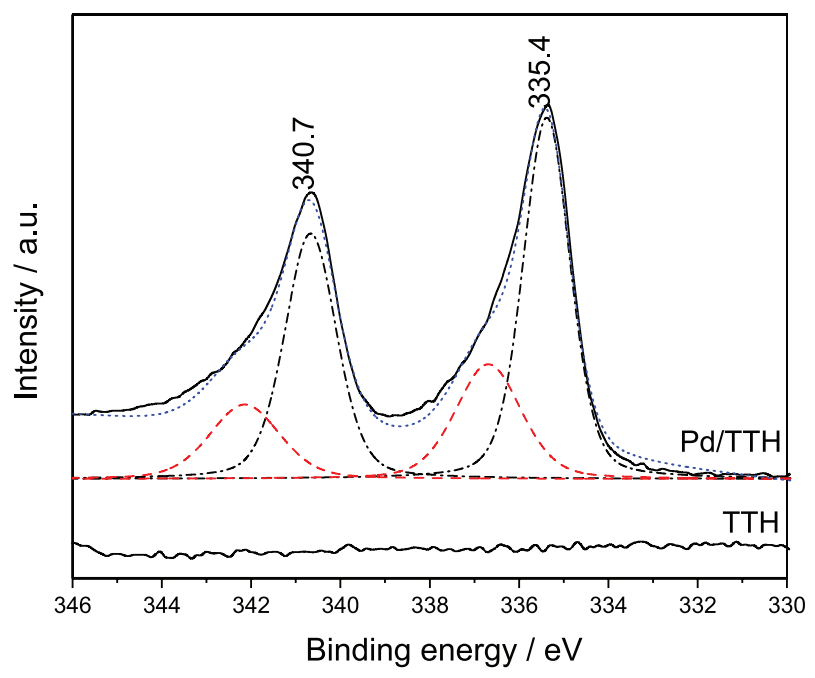

Figure 5. High-resolution XPS spectra at Pd (3d) region for TTH and $\mathrm{Pd} / \mathrm{TTH}$ powder samples. Dashed curves were obtained by deconvolution process; blue line is the resulting sum line from black and red single bands.

Catalytic performance of $\mathrm{Pd} / \mathrm{TTH}$ towards nitrobenzene reduction

The catalytic reduction of nitrobenzene (NB) is commonly used to manufacture aniline (AN), which is an important intermediate for polyurethanes, dyes, pharmaceuticals, explosives, and agricultural products. In this reaction, several poisonous intermediates such as nitrosobenzene, $\mathrm{N}$-phenylhydroxylamine, azoxybenzene, azobenzene, and hydrazobenzene can be formed along with the desired AN product (Figure 6a). The formation and accumulation of these undesired intermediates should be avoided for the green production of AN. ${ }^{40}$ Catalysts based on Pd are known to be capable of selectively hydrogenating nitro-compounds to their corresponding aniline, ${ }^{41,42}$ thus it was investigated the catalytic performance of Pd/TTH by testing it as catalyst for NB reduction and the kinetics of its conversion to AN was accompanied through UV-Vis spectroscopy (Figure 6b).

The catalytic reduction of nitrobenzene in the presence of $\mathrm{NaBH}_{4}$ starts with a reaction between $\mathrm{NaBH}_{4}$ and water at room temperature, yielding $\mathrm{H}_{2}$ and sodium metaborate $\left(\mathrm{NaBO}_{2}\right)$. In the presence of Pd nanoparticles, the $\mathrm{H}-\mathrm{H}$ bond in $\mathrm{H}_{2}$ cleaves and each hydrogen atom attaches to the metal nanoparticle surface, forming metal-hydrogen bonds. The negatively charged hydrogen in the $\mathrm{Pd}-\mathrm{H}$ structure can easily attack the positively charged nitrogen in the nitro group of nitrobenzene, probably adsorbed on the catalyst surface through interactions between the carbonaceous structure and the aromatic nitrobenzene chain. ${ }^{43,44}$ As a result, the nitro group is reduced to the nitroso group, followed by the reductive addition of two hydrogen atoms to form hydroxylamine. Finally, the hydroxylamine is further reduced to the aniline derivative. ${ }^{45}$

It is possible to observe in Figure $6 \mathrm{~b}$ that after $3 \mathrm{~h}$ of reaction, only $38.2 \mathrm{ppm}$ of NB remains (which corresponds to $62 \%$ less than the initial concentration) with the production of $41.34 \mathrm{ppm}$ of AN in solution (96\% of selectivity). It was not possible to observe the formation of other undesirable intermediates, with high selectivity for aniline. This process is of great industrial interest.

To evaluate the stability of $\mathrm{Pd} / \mathrm{TTH}$, reuse tests were performed (Figure 7). After the complete reaction, the catalyst was recovered by centrifugation and reused without 


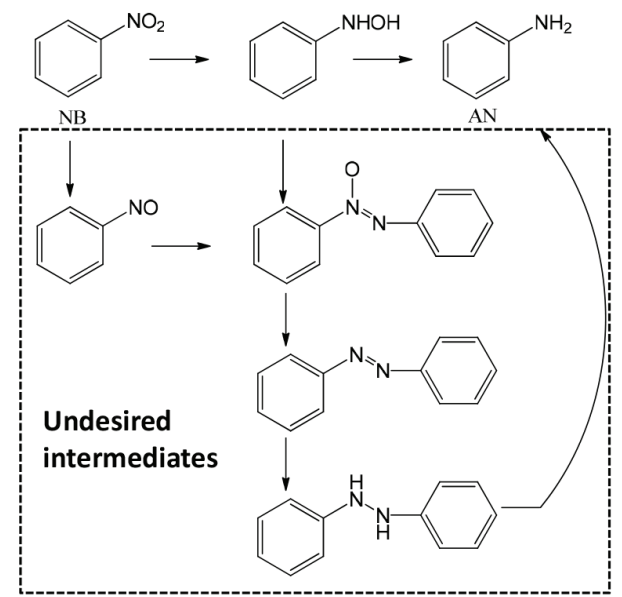

(a)

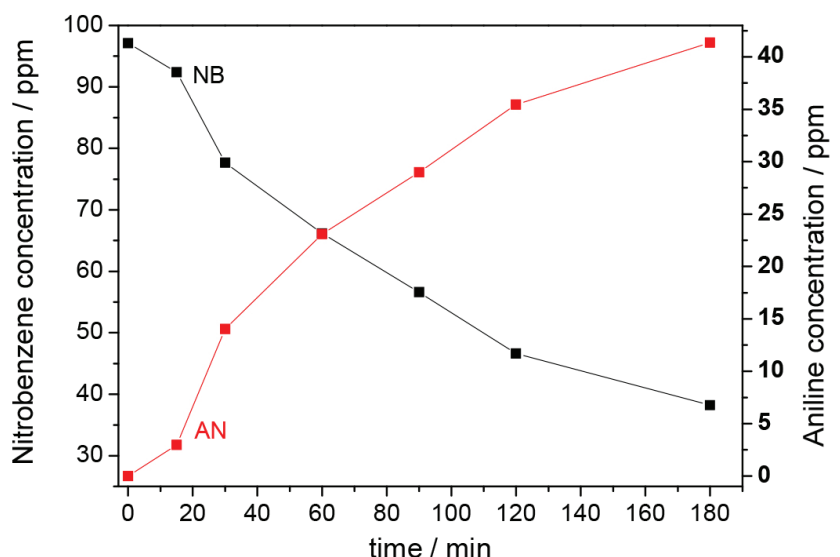

(b)

Figure 6. (a) Possible reaction pathways for the reduction of nitrobenzene. NB: nitrobenzene; AN: aniline; (b) NB (black curve) and AN (red curve) concentrations during NB reduction using Pd/TTH as catalyst in aqueous solution at $25^{\circ} \mathrm{C}$.

any treatment. Reuse tests showed, during four consecutive cycles, $62,60,50$, and $37.5 \%$ of NB removal. These results are very good and demonstrate the stability of Pd/TTH, even with a slight difficulty to separate from the medium due to its small particle size.

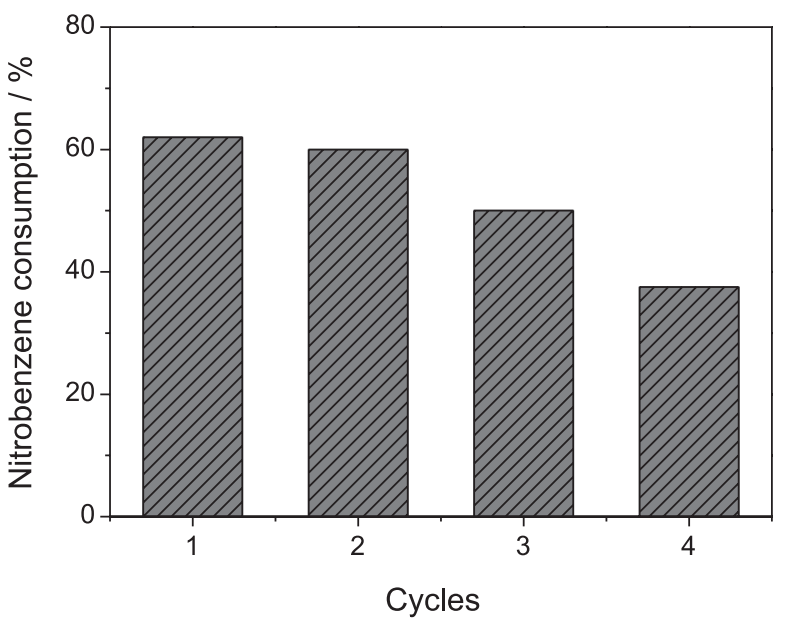

Figure 7. Reuse studies for NB reduction in the presence of $\mathrm{Pd} / \mathrm{TTH}$ material.

The catalytic activity of Pd/TTH towards nitrobenzene reduction to aniline proved to be very similar to other $\mathrm{Pd} / \mathrm{C}$ (palladium on carbon) catalysts reported in literature, considering the different conditions of reaction, ${ }^{45,46}$ with the advantage of using small amounts of $\mathrm{NaBH}_{4}$. These preliminary results show that $\mathrm{Pd} / \mathrm{TTH}$ material has the potential to be applied as Pd-based catalyst for different reactions.

\section{Conclusions}

Human hair was successfully used as adsorbent of $\mathrm{Pd}^{2+}$ in aqueous solution, presenting nearly $100 \%$ of adsorption under the experimental conditions. The pyrolysis of hair fibers containing $\mathrm{Pd}^{2+}$ was performed at $200^{\circ} \mathrm{C}$, preventing the agglomeration of reduced PdNPs. This low-temperature pyrolysis did not cause the complete degradation of amino acid groups present in hair fibers, as shown by FTIR and Raman spectroscopies, besides elemental analysis. TEM and XPS analyses confirmed the presence of PdNPs in the synthesized material with particle sizes below $9 \mathrm{~nm}$, showing that the method used for the reduction of $\mathrm{Pd}^{2+}$ was effective for the formation of well-dispersed nanoparticles. The catalytic performance of this material was studied towards nitrobenzene reduction to aniline in aqueous medium, showing good yields and high selectivity.

\section{Supplementary Information}

Supplementary information is available free of charge at http://jbcs.sbq.org.br and presents the discoloration of the $\mathrm{Pd}^{2+}$ solution after contact with hair fibers (Figure $\mathrm{S} 1$ ), the TG curve obtained for Pd/TTH under air atmosphere (Figure S2), and additional TEM images of Pd/TTH (Figure S3).

\section{Acknowledgments}

The authors thank the Conselho Nacional de Desenvolvimento Científico e Tecnológico (CNPq), Coordenação de Aperfeiçoamento de Pessoal de Nível Superior (CAPES), Fundação do Amparo à Pesquisa do Estado de Minas Gerais (FAPEMIG), and RMQ-MG for financial support. The authors also thank UFMG Microscopy Center for the images obtained. CEM-UFABC 
is also acknowledged for the use of Raman, FT-Raman, and XPS equipment. F. G. M. and W. D P. thank R. M. Lago for fruitful discussions.

\section{References}

1. Boulos, R. A.; Eroglu, E.; Chen, X.; Scaffidi, A.; Edwards, B. R.; Toster, J.; Raston, C. L.; Green Chem. 2013, 15, 1268.

2. Zhao, Z. Q.; Xiao, P. W.; Zhao, L.; Liu, Y. W.; Han, B. H.; RSC Adv. 2015, 5, 73980 .

3. Yang, F.-C.; Zhang, Y.; Rheinstädter, M. C.; PeerJ 2014, $2,619$.

4. Wortmann, F. J.; Wortmann, G.; Schulze zur Wiesche, E.; Langmuir 2010, 26, 7365.

5. Chung, I.-M.; Gopiraman, M.; React. Kinet., Mech. Catal. 2017, 122, 205.

6. Liu, X. J.; Zhou, W. J.; Yang, L. J.; Li, L. G.; Zhang, Z. Y.; Ke, Y. T.; Chen, S. W.; J. Mater. Chem. A 2015, 3, 8840.

7. Sun, G. Q.; Zhou, L. H.; Li, J. X.; Tang, J.; Wang, Y.; RSC Adv. 2015, 5, 71980 .

8. Qian, W. J.; Sun, F. X.; Xu, Y. H.; Qiu, L. H.; Liu, C. H.; Wang, S. D.; Yan, F.; Energy Environ. Sci. 2014, 7, 379.

9. Pramanick, B.; Cadenas, L. B.; Kim, D. M.; Lee, W.; Shim, Y. B.; Martinez-Chapa, S. O.; Madou, M. J.; Hwang, H.; Carbon 2016, 107, 872.

10. Tan, Y. L.; Gao, Q. M.; Yang, C. X.; Yang, K.; Tian, W. Q.; Zhu, L. H.; Sci. Rep. 2015, 5, 12382.

11. Deng, D.; Kim, B. S.; Gopiraman, M.; Kim, I. S.; RSC Adv. 2015, 5, 81492 .

12. Carvalho, A. S. C.; Santos, A. S.; Pereira, S. F. P.; Alves, C. N.; J. Braz. Chem. Soc. 2009, 20, 1153.

13. Ha, B. J.; Kim, S. M.; Kim, C. R.; Jung, O. S.; Bull. Korean Chem. Soc. 2010, 31, 1459.

14. Asubiojo, O. I.; Ajelabi, O. B.; Toxicol. Environ. Chem. 2009, $91,883$.

15. Krishnan, S. S.; Cancilla, A.; Jervis, R. E.; Sci. Total Environ. 1988, 68, 267.

16. Roberts, D. J.; Green, P.; Sci. Total Environ. 1985, 42, 207.

17. Deng, D.; Gopiraman, M.; Kim, S. H.; Chung, I. M.; Kim, I. S.; ACS Sustainable Chem. Eng. 2016, 4, 5409.

18. Gopiraman, M.; Deng, D.; Zhang, K. Q.; Kai, W.; Chung, I. M.; Karvembu, R.; Kim, I. S.; Ind. Eng. Chem. Res. 2017, 56, 1926.

19. Das, S.; Meena, S. S.; Pramanik, A.; J. Colloid Interface Sci. 2016, 462, 307.

20. Gopiraman, M.; Chung, I.-M.; Korean J. Chem. Eng. 2017, 34 , 2169.

21. Gopiraman, M.; Saravanamoorthy, S.; Chung, I.-M.; Res. Chem. Intermed. 2017, 43, 5601.

22. Haveli, S. D.; Walter, P.; Patriarche, G.; Ayache, J.; Castaing, J.; Van Elslande, E.; Tsoucaris, G.; Wang, P. A.; Kagan, H. B.; Nano Lett. 2012, 12, 6212.
23. Mendonca, F. G.; da Cunha, I. T.; Soares, R. R.; Tristao, J. C.; Lago, R. M.; Bioresour. Technol. 2017, 246, 28.

24. Zhong, H.; Su, Y.; Cui, C.; Zhou, F.; Li, X.; Wang, R.; ACS Sustainable Chem. Eng. 2017, 5, 8061.

25. Gavia, D. J.; Shon, Y.-S.; Langmuir 2012, 28, 14502.

26. Thakur, V.; Kumar, S.; Das, P.; Catal. Sci. Technol. 2017, 7, 3692.

27. Zhao, M.; Ji, Y.; Wang, M.; Zhong, N.; Kang, Z.; Asao, N.; Jiang, W.-J.; Chen, Q.; ACS Appl. Mater. Interfaces 2017, 9, 34804.

28. Nassor, E. C. O.; Tristao, J. C.; Oliveira, H. S.; Moura, F. C. C.; dos Santos, E. N.; Lago, R. M.; Araujo, M. H.; Catal. Lett. 2015, 145, 505.

29. Liu, W.; Xu, L.; Lu, G.; Zhang, H.; ACS Sustainable Chem. Eng. 2017, 5, 1368.

30. Zhang, J.; Wang, L.; Shao, Y.; Wang, Y. Q.; Gates, B. C.; Xiao, F. S.; Angew. Chem., Int. Ed. 2017, 56, 9747.

31. Hierso, J. C.; Beauperin, M.; Meunierll, P.; Eur. J. Inorg. Chem. 2007, 24, 3767.

32. Parreira, H. C.; J. Colloid Interface Sci. 1980, 75, 212.

33. Fan, X. Q.; Zhang, L. X.; Zhang, G. B.; Shu, Z.; Shi, J. L.; Carbon 2013, 61, 423.

34. Sun, D.; Ban, R.; Zhang, P. H.; Wu, G. H.; Zhang, J. R.; Zhu, J. J.; Carbon 2013, 64, 424.

35. Peng, H.; Travas-Sejdic, J.; Chem. Mater. 2009, 21, 5563.

36. Movasaghi, Z.; Rehman, S.; Rehman, I. U.; Appl. Spectrosc. Rev. 2007, 42, 493.

37. Madejova, J.; Komadel, P.; Clays Clay Miner. 2001, 49, 410.

38. Singha, S.; Sahoo, M.; Parida, K. M.; Dalton Trans. 2011, 40, 7130 .

39. Zhou, W. P.; Lewera, A.; Larsen, R.; Masel, R. I.; Bagus, P. S.; Wieckowski, A.; J. Phys. Chem. B 2006, 110, 13393.

40. Meng, X.; Cheng, H.; Akiyama, Y.; Hao, Y.; Qiao, W.; Yu, Y.; Zhao, F.; Fujita, S.-i.; Arai, M.; J. Catal. 2009, 264, 1.

41. Sun, J.; Fu, Y.; He, G.; Sun, X.; Wang, X.; Catal. Sci. Technol. 2014, 4, 1742.

42. Souza, F. D.; Fiedler, H.; Nome, F.; J. Braz. Chem. Soc. 2016, 27,372 .

43. Xiong, P.; Chen, Q.; He, M.; Sun, X.; Wang, X.; J. Mater. Chem. 2012, 22, 17485.

44. Zuo, G.; Zhou, X.; Huang, Q.; Fang, H.; Zhou, R.; J. Phys. Chem. C 2011, 115, 23323.

45. Nasrollahzadeh, M.; Sajadi, S. M.; Rostami-Vartooni, A.; Alizadeh, M.; Bagherzadeh, M.; J. Colloid Interface Sci. 2016, $466,360$.

46. Kim, Y.; Ma, R.; Reddy, D. A.; Kim, T. K.; Appl. Surf. Sci. 2015 , $357,2112$.

Submitted: August 9, 2018

Published online: October 1, 2018 